\title{
Morphology Induced Spinodal Decomposition at the Surface of Symmetric Diblock Copolymer Films
}

\author{
Robert D. Peters, ${ }^{\dagger}$ Pawel Stasiak, ${ }^{\ddagger}$ Mark W. Matsen, ${ }^{\ddagger}$ and Kari Dalnoki-Veress ${ }^{*}, \dagger, \S$ \\ ${ }^{\dagger}$ Department of Physics and Astronomy, McMaster University, Hamilton, Ontario, Canada \\ ${ }^{\ddagger}$ School of Mathematical and Physical Sciences, University of Reading, Whiteknights, Reading, United Kingdom \\ ${ }^{\S}$ Laboratoire de Physico-Chimie Theorique, UMR CNRS, Gulliver 7083, ESPCI, Paris, France
}

\section{Supporting Information}

ABSTRACT: Atomic force microscopy is used to study the ordering dynamics of symmetric diblock copolymer films. The films order to form a lamellar structure which results in a frustration when the film thickness is incommensurate with the lamellae. By probing the morphology of incommensurate films in the early ordering stages, we discover an intermediate phase of lamellae arranged perpendicular to the film surface. This morphology is accompanied by a continuous growth in amplitude of the film surface topography with a characteristic wavelength, indicative of a spinodal process. Using selfconsistent field theory, we show that the observation of perpendicular lamellae suggests an intermediate state with parallel lamellae at the substrate and perpendicular lamellae at
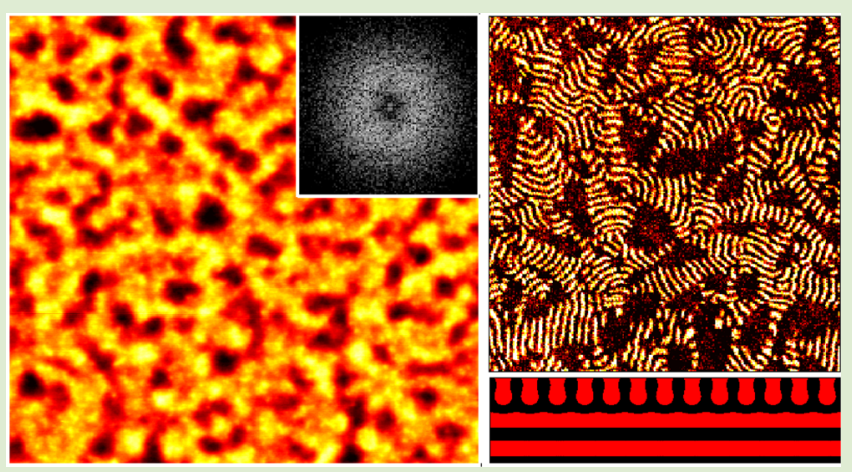

the free surface. The calculations confirm that the intermediate state is unstable to thickness fluctuations, thereby driving the spinodal growth of surface structures.

S pinodal processes involve the continuous and rapid growth of two or more distinct phases from an unstable initial state, as opposed to the slower nucleation processes when the initial state is metastable. Spinodal dynamics are known to occur in a wide array of physical systems including alloys, ${ }^{1}$ binary fluids, ${ }^{2}$ glasses, ${ }^{3}$ liquid-crystalline films, ${ }^{4}$ polymer blends, ${ }^{2}$ dewetting polymer films, ${ }^{5-9}$ and even the rupture of foams in a champagne glass. ${ }^{10}$ Thin films of symmetric diblock copolymer are of particular interest ${ }^{11-13}$ because they are a simple 2D system where the dynamics of the conserved order parameter $\Psi(x, y)=h(x, y)-h_{0}$ (film thickness relative to the initial uniform thickness) are potentially slow enough to observe the early time behavior. ${ }^{14}$ Maaloum et al. first surmised the existence of a spinodal process in diblock copolymer films from the long-time real-space patterns of $\Psi(x, y)$, which looked as if they emerged from fluctuations of a single characteristic wavelength. ${ }^{11}$ They therefore assumed that the initial disordered state is unstable with respect to variations in film thickness. Joly et al. provided further evidence for a spinodal process by the absence of an incubation time. ${ }^{12}$ However, they proposed a different explanation, which assumes that the free energy of an ordered film, $F(\Psi)$, is a smooth function with an interval of negative curvature (i.e., unstable region) between the two stable film thicknesses. Although these studies have speculated the existence of a spinodal process, conclusive proof and a solid theoretical explanation have yet to be provided. There is no reason for the disordered film to be unstable, nor is the free energy of ordered films a smooth continuous function of film thickness.

In an "AB" diblock copolymer, incompatibility between the two blocks is characterized by the Flory-Huggins parameter, $\chi$, which varies inversely with temperature. When the blocks segregate, like oil and water, it is the relative size of the A block to the entire polymer chain, $f$, that determines the morphology. Symmetric diblock copolymers, with equally sized blocks $(f=$ $1 / 2$ ), assemble into a stack of $\mathrm{AB} / \mathrm{BA}$ bilayers creating a lamellar phase of alternating $\mathrm{A}$ - and B-rich domains. In the bulk, this ordered periodic microstructure forms from the disordered melt once $\chi N \gtrsim 10,{ }^{15,16}$ where $N$ is the total polymerization index of the copolymers. The preferred thickness of a bilayer, $L_{0}$, is determined by a competition between the entropic cost of stretching polymers and the tension of the $\mathrm{A}-\mathrm{B}$ interfaces. ${ }^{17}$

Though equilibrium morphologies of diblock copolymers in the bulk are well understood, ${ }^{15-17}$ the study of these molecules under confinement is of particular interest owing to their potential for use in a variety of applications and for providing insight into physics at the nanoscale. ${ }^{18-22}$ In the case of thin films supported on a substrate, the copolymer is confined in 1dimension. The difference in interfacial energies of the $A$ and $B$ block with the substrate and the free (air or vacuum) surface

Received: April 2, 2013

Accepted: April 30, 2013 
can cause preferential segregation of a particular block at each interface. In the case of symmetric diblocks, these preferred surface interactions direct lamellae to orient parallel to the interfaces. ${ }^{23-28}$ To preserve the equilibrium bilayer thickness throughout the film, the film thickness at equilibrium must satisfy the commensurability condition $h=L_{0} \nu / 2$, where $\nu$ is an integer denoting the number of $\mathrm{AB}$ monolayers. We denote this parallel phase $\mathrm{L}_{v}^{\|}$. For symmetric wetting conditions where both interfaces have an affinity for the same block, $\nu$ is even. Conversely, for asymmetric wetting, $\nu$ must be odd. Films with initial thickness, $h_{0}$, which do not satisfy the quantized commensurability condition, will order into regions consisting of $\nu$ and $\nu+2$ monolayers, with a step of thickness $L_{0}$ separating the $\mathrm{L}_{v}^{\|}$and $\mathrm{L}_{v+2}^{\|}$regions. With increasing $h_{0}$, the areal fraction of the $L_{v+2}^{\|}$phase expands to satisfy volume conservation. In this case, the relief structures evolve from islands, to a bicontinuous pattern, to holes when the majority of the film is $\mathrm{L}_{v+2}^{\|}{ }^{23,24}$ Though these structures have been well studied, direct measurements of film morphologies during the intermediate stages of annealing in symmetric diblock films remain elusive, yielding a gap in our understanding of the ordering process.

Ordering of these lamellar forming films is known to initiate at the interfaces. ${ }^{26,28-30}$ Once the microphase separation of the diblocks is complete within the film, the topography of the free surface begins to develop relief structures by either nucleation $^{13,31-33}$ or spinodal ${ }^{1-13}$ growth. Though nucleated growth has been shown to arise in nearly commensurate films from a metastable state consisting of parallel lamellae with nonequilibrium bilayer thickness, ${ }^{32,33}$ an explanation for the observation of spinodal decomposition of topological structure far from commensurability is yet to be provided. In order to investigate the origins of the spinodal morphology, we focus here on the pathway as the film transitions from a homogeneous slab to the final ordered phase during sample annealing. We will show that there is an intermediate morphology at the free surface that mediates the transition, and we establish the link between this morphology and the subsequent spinodal decomposition of the topography. Selfconsistent field theory (SCFT) calculations verify the differences in the free energy of the observed structures that drive spinodal decomposition.

Experiments were performed using poly(styrene-methyl methacrylate) (PS-PMMA) copolymer, an asymmetrically wetting diblock. The topography evolution of an incommensurate $157 \mathrm{~nm}(\nu \approx 12)$ film annealed at $155{ }^{\circ} \mathrm{C}$ is shown in Figure 1. The upper panels show the spinodal growth of surface

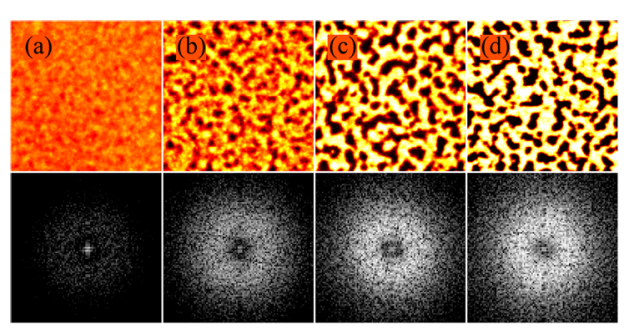

Figure 1. (Top Row) AFM topography images of $157 \mathrm{~nm}(\nu \approx 12)$ film for annealing times of (a) $t=175$, (b) $t=265$, (c) $t=355$, and (d) $t=445 \mathrm{~min}$ at $155^{\circ} \mathrm{C}$. Scan size is $25 \mu \mathrm{m}$. The vertical scale is the same for each image with black to white corresponding to a $30 \mathrm{~nm}$ thickness difference. (Bottom Row) 2DFT obtained from each AFM image. relief structures typical of all incommensurate films studied, evolving from initial undulations of $<3 \mathrm{~nm}$ at annealing times of $t=175 \mathrm{~min}$ to equilibrium fluctuations of $26.5 \mathrm{~nm}$ corresponding to the full bilayer thickness at $t=445 \mathrm{~min}$. The lower panels plot the 2D Fourier transform (2DFT) computed at each annealing stage. The spinodal ring, which grows in intensity for longer annealing times, is clearly visible. From the radial average of the 2DFT, we obtain a peak frequency of the spinodal pattern at $\sim 0.3 \mu \mathrm{m}^{-1}$, corresponding to a correlation wavelength of $\sim 3 \mu \mathrm{m}$ (Figure $2 \mathrm{a}$ ). Though we
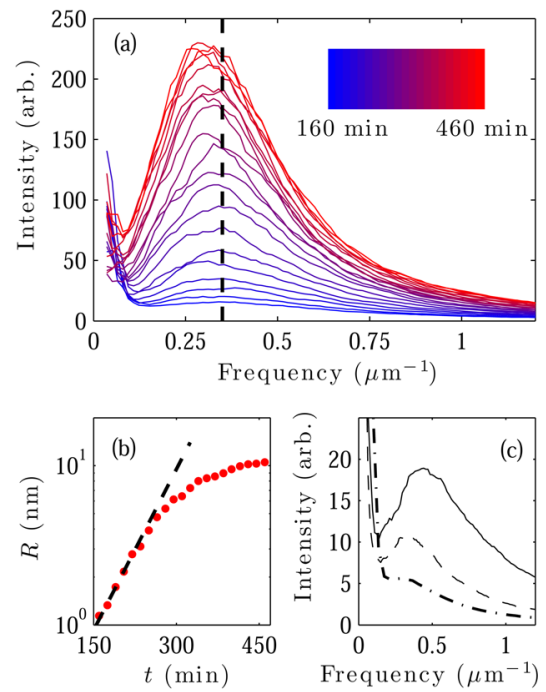

Figure 2. (a) Radial average 2DFT of AFM topography images for increasing annealing times. Dashed line is average peak position at early annealing times. (b) Root-mean-squared roughness, $R$, as a function of time on a log-linear plot. Straight dashed line shows the exponential growth of the thickness fluctuations at early annealing times. (c) Radial average of the 2DFT for films with initial thickness $129 \mathrm{~nm}$ (solid line), $157 \mathrm{~nm}$ (dashed line), and $187 \mathrm{~nm}$ (dasheddotted line), corresponding to $\nu \approx 10,12$, and 14 respectively.

only quantify the early stages of the topography evolution in accordance with the linearized spinodal theory, we do also observe the onset of coarsening through a shift in the 2DFT peak toward shorter frequencies (see Supporting Information).

The root-mean-squared roughness, $R$, is calculated to characterize the growth of surface fluctuations. ${ }^{12,31}$ In the early stages of spinodal growth, the film exhibits an exponential growth rate in $R$, consistent with a spinodal process (Figure $2 b){ }^{2}$ As annealing continues, the roughness plateaus as the equilibrium lamellar thickness is reached. The 2DFT peak amplitude also displays these growth kinetics. ${ }^{34}$ In addition to the measurements of a film with $\nu \approx 12$, the measurement of early stage spinodal growth was observed for incommensurate films with initial thickness of $h_{0} \approx 10 L_{0} / 2$ and $h_{0} \approx 14 L_{0} / 2$. These films vary in thickness by about $60 \mathrm{~nm}$, yet all exhibit similar spinodal wavelengths of $2-3 \mu \mathrm{m}$, with a slight increase in wavelength for thicker films (Figure 2c). The similarity in wavelength suggests that the driving force is dependent primarily on the film commensurability and not total film thickness: the wavelength is a property of the diblock structure nearest to the free surface.

As spinodal fluctuations begin to form $(t \sim 160 \mathrm{~min})$, we probe the morphology at the film surface. An AFM phase image taken during the onset of spinodal growth (Figure 3 ) clearly shows a majority of lamellae arranged perpendicular to the film 

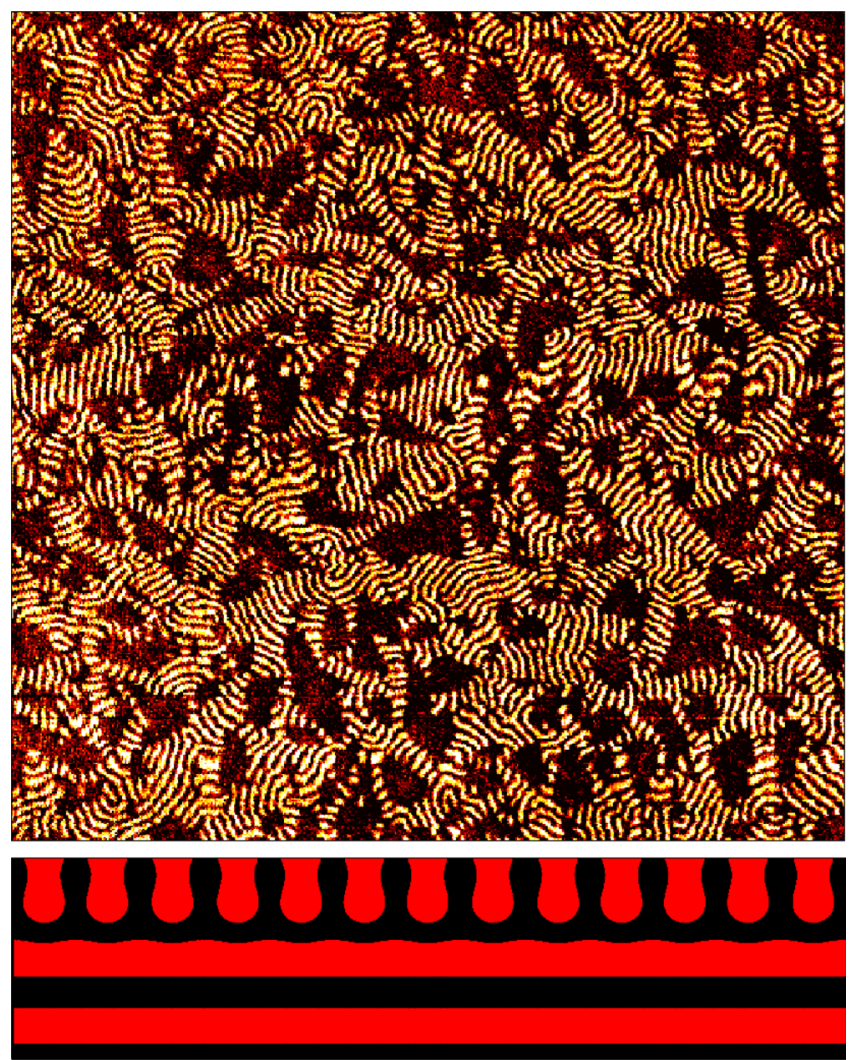

Figure 3. (Top) Phase image $(2.5 \mu \mathrm{m})$ of film surface at $t \sim 160 \mathrm{~min}$ showing majority perpendicular lamellar morphology (PS dark, PMMA light). (Bottom) SCFT calculation of mixed morphology intermediate state.

surface. The perpendicular lamellae of this intermediate morphology have the expected equilibrium bilayer period, $L_{0}$. The larger dark areas in the phase image indicate regions of non-perpendicular morphology caused by the growth of parallel lamellae. This rearrangement is facilitated by the spinodal topography fluctuations at the surface that are already detectable at these annealing times, as demonstrated in Figure 1. Films that are closer to commensurate show nucleated growth of islands or holes, without passing through the intermediate perpendicular lamellar phase of Figure $3{ }^{33}$ Thus, the intermediate morphology that facilitates spinodal decomposition into the $L_{v}^{\|}$and $L_{v+2}^{\|}$regions is a feature of the frustration in thin maximally incommensurate films.

For all maximally incommensurate films studied, we always observed perpendicular lamellae at the film surface preceding the spinodal growth in the surface topography. As a consequence, our measurements indicate that, for highly incommensurate films, an intermediate ordered state must consist of either (i) perpendicular lamellae throughout the entire film or (ii) a mixed morphology state consisting of perpendicular lamellae at the free surface and parallel lamellae at the substrate, as shown in Figure 3. The PMMA block is known to have a strong preferential interaction with the $\mathrm{Si}$ substrate. $^{29,35}$ This surface affinity has been shown to align lamellae parallel to the substrate, well above the ODT, ${ }^{29,30}$ and consequently, a parallel morphology will exist near the substrate upon annealing. Therefore, the perpendicular lamellae at the free surface of incommensurate films indicate that a mixed morphology must occur as an intermediate ordering state. Mixed morphologies have been observed in films with a neutral substrate $^{36}$ and in capped films; ${ }^{37}$ however, the existence of a mixed morphology as an intermediate state in the ordering of films with a free surface is new.

To gain further insight into our system, Figure 4 shows the free energies, $F$, of different morphologies calculated with

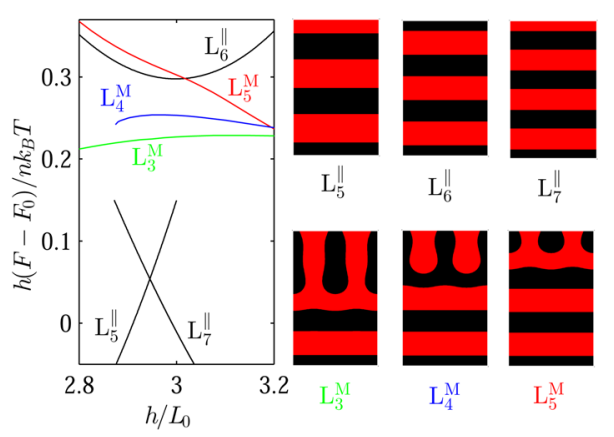

Figure 4. Free energy, $F$, of parallel $\left(\mathrm{L}_{v}^{\|}\right)$and mixed $\left(\mathrm{L}_{v}^{\mathrm{M}}\right)$ lamellar morphologies plotted as a function of film thickness, $h . F_{0}$ is the free energy of the bulk lamellar phase.

SCFT. Our calculations follow ref 25 , except that we use reflecting boundary conditions at the substrate and air surfaces. ${ }^{38}$ The experimental conditions are approximated by setting the block copolymer parameters to $\chi N=25$ and $f=0.5$ and providing an affinity for PS (red) at the air surface of strength $\Lambda N=0.3$, as defined in ref 38 . The substrate affinity for PMMA is much greater, which rules out the possibility of perpendicular lamellae according to previous SCFT calculations. ${ }^{38}$ Therefore, we only consider morphologies where the entire substrate is coated by PMMA (black). We chose a film thickness of $h \approx 6 L_{0} / 2(\nu \approx 6)$, as thicker films, like those used in our experimental study, will differ only in the number of parallel layers near the substrate. A film thickness corresponding to $\nu \approx 6$ is suited to the $\mathrm{L}_{6}^{\|}$morphology, but nevertheless, the $L_{5}^{\|}$or $L_{7}^{\|}$morphologies of either stretched or compressed domains are energetically favorable because they allow for the exposure of PS at the air surface. The mixed $\left(\mathrm{L}_{v}^{\mathrm{M}}\right)$ morphologies allow all lamellae to adopt the preferred domain thickness while still allowing a majority of the air surface to be covered by PS. However, there is still some PMMA at the air surface as well as a grain boundary between the parallel and perpendicular lamellae, making mixed morphologies less stable than the $L_{5}^{\|}$and $L_{7}^{\|}$morphologies. Notice that the free energies of $L_{5}^{\|}$and $L_{7}^{\|}$do not form a single smooth curve as originally assumed by Joly et al. ${ }^{12}$

Although the SCFT tells us which morphologies are favored at equilibrium, one has to consider the kinetics involved in forming the domains. The substrate has a far stronger affinity for PMMA than the air surface does for PS, ${ }^{29}$ thus, the ordering will begin at the substrate with parallel lamellae of the preferred thickness. As the disordered region near the air interface gets thinner, the incommensurability of the film will become apparent. If the system continues to form parallel monolayers of equilibrium thickness, the system will end up in the costly $\mathrm{L}_{6}$ morphology, with PMMA at the free interface. However, in order to form the more favorable $\mathrm{L}_{5}^{\|}$or $\mathrm{L}_{7}^{\|}$morphologies, the system must adjust the thickness of the existing lamellae near the substrate, which is a slow process due to the necessity of polymer exchange between layers. ${ }^{39}$ The alternative is for the remaining disordered region to form perpendicular lamellae, resulting in the mixed morphology $\mathrm{L}_{4}^{\mathrm{M}}$ structure. The $\mathrm{L}_{5}^{\mathrm{M}}$ 
morphology is unfavorable because of the high free energy associated with the excess bending of the lamellar domains. Despite the lower free energy of the $\mathrm{L}_{3}^{\mathrm{M}}$ morphology, we expect this to be bypassed because of the rapid formation of parallel lamellae; it is only when there is a disordered layer of thickness $\sim L_{0}$ that the frustration of incommensurability is apparent to the system.

With the system in the intermediate morphology, $\mathrm{L}_{4}^{\mathrm{M}}$, the subsequent evolution of the system is then intimately related to the curvature of the free energy. The negative curvature of the $\mathrm{L}_{4}^{\mathrm{M}}$ morphology implies that it is unstable with respect to small variations in film thickness. ${ }^{25}$ Consequently, any flow of material from one area of the film to another will lower the overall free energy of the system; this instability is the key ingredient for spinodal fluctuations to occur.

Direct measurement of an unstable intermediate mixed morphology for highly incommensurate films completes the picture of symmetric diblock copolymer film ordering. As has already been well established, surface relief structures grow via a nucleated process for nearly commensurate films due to a metastable intermediate state of deformed parallel lamellae. ${ }^{32,33}$ Conversely, the maximally incommensurate films will exhibit spinodal growth patterns induced by a kinetically favored unstable intermediate state of mixed lamellar orientations. Additionally, in the regime of moderate incommensurability we might expect that nucleated and spinodal growth kinetics could occur simultaneously, as is indeed observed in experiment (see Supporting Information).

In conclusion, we have characterized the ordering process of PS-PMMA symmetric copolymer thin films. Identifying a direct link between our observations of an intermediate film morphology and surface relief growth kinetics, we have determined the source of spinodal decomposition in the film topography. The ordering pathway consists of an intermediate state of mixed lamellar morphology which precedes topological fluctuations. The free energy of the mixed morphology is calculated using SCFT, and is found to be an unstable state. In particular, it has a negative curvature in the free energy as a function of film thickness, providing the driving force necessary for the spinodal decomposition of the surface fluctuations. These results provide a clear picture of the ordering pathway of symmetric diblock films through an intermediate mixed morphology that is unstable to spinodal thickness variations.

\section{EXPERIMENTAL SECTION}

PS-PMMA copolymer of molecular weight for the blocks $M_{\mathrm{w}}(\mathrm{PS}) \approx$ $25 \mathrm{~kg} / \mathrm{mol}, M_{\mathrm{w}}(\mathrm{PMMA}) \approx 26 \mathrm{~kg} / \mathrm{mol}$, and dispersity $=1.06$ (Polymer Source, Canada) was used. The equilibrium bilayer thickness was determined to be $L_{0}=26.5 \mathrm{~nm}$ using atomic force microscopy (AFM; Veeco Caliber, U.S.A.). Samples were spincast in air from dilute toluene solutions onto $\mathrm{Si}$ substrates with the native oxide layer present, such that they were maximally incommensurate, that is, $h_{0} \approx L_{0} \nu / 2$ with $\nu$ even, because PS-PMMA is asymmetrically wetting. The initial ascast films exhibit thickness variations of less than $1 \mathrm{~nm}$ as measured using ellipsometry and have no long-range order due to rapid solvent evaporation during spincoating. These films were subsequently annealed at $155{ }^{\circ} \mathrm{C}$, well below the order-disorder transition temperature (ODT), causing the film to order into lamellae. The samples were monitored during annealing using an ellipsometry technique sensitive to changes in film morphology, ${ }^{33}$ and quenched into the glassy state as soon as surface topography began to change. Using AFM in tapping mode, the film surface was studied both with topography and phase imaging. The phase signal is sensitive to the mechanical contrast between PS and PMMA domains at the surface. ${ }^{40}$ We continue to anneal films at $155^{\circ} \mathrm{C}$, interrupted by rapid quenches into the glassy state to observe the evolution of the film surface with AFM.

\section{ASSOCIATED CONTENT}

\section{S Supporting Information}

(i) Analysis of 2DFT peak amplitude and position revealing the onset of coarsening of the surface topography at large times. (ii) Topography evolution of films with moderate incommensurability demonstrating both metastable and spinodal processes. This material is available free of charge via the Internet at http://pubs.acs.org.

\section{AUTHOR INFORMATION}

\section{Corresponding Author}

*E-mail: dalnoki@mcmaster.ca.

\section{Notes}

The authors declare no competing financial interest.

\section{ACKNOWLEDGMENTS}

Financial Support for this work was provided by NSERC (Canada) and EPSRC (U.K.).

\section{REFERENCES}

(1) Gerold, V.; Kostorz, G. J. Appl. Crystallogr. 1978, 11, 376-404.

(2) Gunton, J. D.; San Miguel, M.; Sahni, P. S. The Dynamics of First Order Phase Transitions. In Phase Transitions and Critical Phenomena; Domb, C., Lebowitz, J. L., Eds.; Academic Press: London, 1983; Vol. 8.

(3) Jantzen, C. M. F.; Herman, H. . In Phase Diagrams: Material Science and Technology; Alpter, A. A., Ed.; Academic Press: New York, 1978; Vol. 5.

(4) Vandenbrouck, F.; Valignat, M. P.; Cazabat, A. M. Phys. Rev. Lett. 1999, 82, 2693-2696.

(5) Redon, C.; Brochard-Wyart, F.; Rondelez, F. Phys. Rev. Lett. 1991, 66, 715-718.

(6) Reiter, G. Phys. Rev. Lett. 1992, 68, 75-78.

(7) Sferrazza, M.; Heppenstall-Butler, M.; Cubitt, R.; Bucknall, D.; Webster, J.; Jones, R. A. L. Phys. Rev. Lett. 1998, 81, 5173-5176.

(8) Higgins, A. M.; Jones, R. A. L. Nature 2000, 404, 476-478.

(9) Seemann, R.; Herminghaus, S.; Jacobs, K. Phys. Rev. Lett. 2000, $86,5534-5537$.

(10) Bergeron, V. J. Phys.: Condens. Matter 1999, 11, R215-R238.

(11) Maaloum, M.; Ausserre, D.; Chatenay, D.; Gallot, Y. Phys. Rev. Lett. 1993, 70, 2577-2580.

(12) Joly, S.; Raquois, A.; Paris, F.; Hamdoun, B.; Auvray, L.; Ausserre, D.; Gallot, Y. Phys. Rev. Lett. 1996, 77, 4394-4397.

(13) Vignaud, G.; Bigaud, A.; Grubel, G.; Joly, S.; Ausserre, D.; Legrand, J. F.; Gallot, Y. Physica B 1998, 248, 250-257.

(14) This representation of the order parameter, $\Psi$, as a height difference is analogous to the use of a compositional order parameter for a two-component blend. The two components of the system are effectively the heights of (i) an island and (ii) a hole; film heights between these two extremes may be regarded as a mixture of the two components.

(15) Bates, F. S. Science 1991, 251, 898-905

(16) Bates, F. S.; Fredrickson, G. H. Annu. Rev. Phys. Chem. 1990, 41, 525-557.

(17) Matsen, M. W. J. Phys: Condens. Matter 2002, 14, R21-R47.

(18) Hamley, I. Prog. Polym. Sci. 2009, 34, 1161-1210.

(19) Thurn-Albrecht, T.; Schotter, J.; Kastle, G. A.; Emley, N.; Schibauchi, T.; Krusen-Ebaum, L.; Guarini, K.; Black, K. T.; Tuominen, M. T.; Russell, T. P. Science 2000, 290, 2126-2129.

(20) Tang, C.; Lennon, E. M.; Fredrickson, G. H.; Kramer, E. J.; Hawker, C. J. Science 2008, 322, 429-432.

(21) Creton, C.; Kramer, E. J.; Brown, H. R.; Hui, C. Y. Adv. Polym. Sci. 2001, 156, 53-136. 
(22) Mansky, P.; Liu, Y.; Huang, E.; Russell, T. P.; Hawker, C. Science 1997, 275, 1458-1460.

(23) Green, P. F.; Limary, R. Adv. Colloid Interface Sci. 2001, 94, 5381.

(24) Fasolka, M. J.; Mayes, A. M. Annu. Rev. Mater. Res. 2001, 31, $323-355$.

(25) Matsen, M. J. Chem. Phys. 1997, 106, 7781-7791.

(26) Shull, K. R. Macromolecules 1992, 25, 2122-2133.

(27) Croll, A. B.; Massa, M. V.; Matsen, M. W.; Dalnoki-Veress, K. Phys. Rev. Lett. 2006, 97, 204502.

(28) Croll, A. B.; Shi, A.-C.; Dalnoki-Veress, K. Phys. Rev. E 2009, 80, 051803.

(29) Anastasiadis, S. H.; Russell, T. P.; Satija, S. K.; Majkrzak, C. F. Phys. Rev. Lett. 1989, 62, 1852-1855.

(30) Menelle, A.; Russell, T. P.; Anastasiadis, S. H.; Satija, S. K.; Marjkrzak, C. F. Phys. Rev. Lett. 1992, 68, 67-70.

(31) Collin, B.; Chatenay, D.; Coulon, G.; Ausserre, D.; Gallot, Y. Macromolecules 1992, 25, 1621-1622.

(32) Joly, S.; Ausserre, D.; Brotons, G.; Gallot, Y. Eur. Phys. J. E 2002, $8,355-363$.

(33) Peters, R. D.; Dalnoki-Veress, K. Eur. Phys. J. E 2012, 35, 132. (34) The peak amplitude in the 2DFT radial average displays the same physical characteristics as the RMS roughness (see Supporting Information). However, due to the complex 2DFT background of the AFM tip and variations between measurements, we find roughness to be a more robust measure.

(35) Fasolka, M. J.; Banerjee, P.; Mayes, A. M.; Pickett, G.; Balazs, A. C. Macromolecules 2000, 33, 5702-5712.

(36) Mansky, P.; Russell, T. P.; Hasker, C. J.; Pitsikalis, M.; Mays, J. Macromolecules 1997, 30, 6810-6813.

(37) Koneripalli, N.; Levicky, R.; Bates, F. S. Langmuir 1996, 12, 6681-6690.

(38) Matsen, M. W. Macromolecules 2006, 39, 5512-5520.

(39) Croll, A. B.; Matsen, M. W.; Shi, A.-C.; Dalnoki-Veress, K. Eur. Phys. J. E 2008, 27, 407-411.

(40) Vu, T.; Mahadevapuram, N.; Perera, G. M.; Stein, G. E. Macromolecules 2011, 44, 6121-6127. 